\title{
Irreversible Electroporation: A Novel Image-Guided Cancer Therapy
}

\author{
Edward W. Lee, Susan Thai, and Stephen T. Kee \\ Division of Interventional Radiology, Department of Radiology, David Geffen School of Medicine at UCLA, Los Angeles, CA, USA
}

Irreversible electroporation (IRE) is a novel tumor ablation technique using a non-thermal energy to create innumerable permanent nanopores in the cell membrane to disrupt cellular homeostasis. This disruption of cellular homeostasis initiates apoptosis which leads to permanent cell death. In our translational research, we have demonstrated that IRE can be a safe, fast and powerful method of tumor treatment. In this review, we present an overview of IRE ablation including a brief history of IRE, advantages and disadvantages of IRE and clinical and research implications of IRE. (Gut Liver 2010;4(Suppl. 1): S99-104)

Key Words: Irreversible electroporation; Apoptosis; Tumor treatment

\section{INTRODUCTION}

With the continual progression of cancer therapy research, each new development refines the approach to treating specific cancer types. While many new methods have introduced important advantages in treating and eliminating tumors, there still exists a high demand for less invasive forms of treatment that can precisely target specific tumors. In particular, a new method called irreversible electroporation (IRE) has recently been shown to be a more precise and complete method of tumor ablation. IRE, a minimally invasive treatment, induces cell death by creating permanent nanopores in the cellular membrane using high voltage direct electrical current. These iatrogenically created nanopores disrupt the cellular homeostasis, resulting in cell death via apoptosis. ${ }^{1-3}$

IRE, with its novel ablation mechanism, provides a more refined approach to eliminating solid tumor without the known complications of other conventional ablative methods. In this review, we present a brief history of IRE, explore the up-to-date research findings, and discuss the effectiveness and limitations of IRE.

\section{EVOLUTION OF IRE}

IRE has been observed as early as 1754 when Nollet studied the release of a static electrical generator on the skin. ${ }^{4}$ Since then, the first article on irreversible electroporation was published in 1898 by Fuller et al. ${ }^{5}$, which reported that multiple high voltage discharges had bactericidal effects on a water sample. In 1903, Rockwell ${ }^{6}$ reported that under the same discharges that Fuller reported, red corpuscles experienced a loss of color and a change of shape, which may have resulted from the hemolysis of water, generated by irreversible electroporation.

Recently, true clinical application of IRE was derived from the utilization of reversible electroporation, a method in which gene uptake into eukaryotic cells are dramatically increased when electrical fields are used to temporarily open membrane pores for gene insertion into cells (electrogenetherapy). ${ }^{7,8}$ During reversible electroporation, the cell death created by irreversible electroporation was considered highly undesirable in the past. However, further investigations on the use of irreversible electroporation as a method of permanently destroying cancerous cells has led to its clinical application in tumor ablation. ${ }^{9}$

The first studies of IRE involved the exploration of cellular destruction through the application of electrical currents. Research conducted by Davalos et al. ${ }^{9}$ tested this concept using a mathematical model and in vitro models.

Correspondence to: Stephen T. Kee, Edward W. Lee

Division of Interventional Radiology, Department of Radiology, Ronald Reagan Medical Center at UCLA, Los Angeles, CA, USA

Tel: +1-310-206-4277, Fax: +1-310-206-1294, E-mail: Skee@mednet.ucla.edu, EdwardLee@mednet.ucla.edu

DOI: $10.5009 /$ gnl.2010.4.S1.S99 
Results from these studies showed that IRE could indeed induce cell death without using thermal energy.

Further studies then sought to optimize the tissue ablation in vivo. Investigations by Edd et al. ${ }^{10}$ demonstrated that areas of ablation produced by irreversible electroporation are clearly defined with cell scale resolution between ablated and non-ablated zones. A later study complemented the previous findings with the conclusion that IRE spared certain areas when used on sensitive organs, such as the collagen network in the urethra. ${ }^{11}$ Furthermore, this study proposed that IRE may administer an immunological response that can aid with the clearing of dead tissues after treatment. Al-Sakere et al. ${ }^{12}$ conducted the first successful in vivo IRE procedure on mice tumors. Optimum results were achieved when a higher number of electrical pulses were delivered, specifically a tenfold increase from the 8 pulses used in most reversible electroporation settings. ${ }^{12}$ Their findings confirmed that IRE, negligible of any heating, was the factor responsible for complete tumor ablation.

With increased interest in IRE as a new tumor ablation method, it was imperative to confirm these findings on a larger animal to verify the potential for clinical application. We have shown that IRE has the potential to ablate large volumes of tissue in a significantly short period of time. Additionally, the electrical parameters used do not stimulate significant thermal effects, even though they are above those normally used in reversible electroporation, in terms of electrical field magnitude, pulse duration, and number of pulses. ${ }^{13}$ Most of the large animal studies have been conducted on normal swine liver tissues ${ }^{2,13}$ and on VX2 tumors implanted in rabbit livers (unpublished data). Currently, over 20 institutions throughout the U.S and internationally are actively involved in pre-clin$\mathrm{ical} /$ translational research of IRE involving liver tumor, ${ }^{2,14,15}$ brain tumor, ${ }^{16}$ breast tumor, ${ }^{17}$ lung tumor, pancreatic tumor and kidney tumors.

Preliminary data of IRE in human clinical trials have been presented at many international conferences over the last two years. Most of them are treatments for hepatocellular carcinoma, hepatic colorectal metastasis, renal cell carcinoma, and primary bronchogenic tumor. However, no data has been published other than a review discussing the unique challenges of anesthesia involving the patients undergoing IRE treatment. ${ }^{18}$

\section{WHY IRE?}

Several unique characteristics of IRE distinguish it from other currently available tumor ablative techniques, such as radiofrequency ablation (RFA), cryoablation (Cryo) or microwave ablation (MWA). These features are based on how IRE produces tumor ablation through non-thermal, electric field-induced mechanisms. Other tumor ablation techniques rely on heat to induce cell death, which can lead to adverse thermal effects.

\section{Short ablation time}

A typical IRE procedure for a solid tumor, with a size of approximately $3 \mathrm{~cm}$ in diameter, uses 90 pulses with an ultra-short pulse length of 100 microseconds. A single IRE ablation session takes less than one minute. Therefore, even with three or four overlapping ablations, total IRE treatment time is under 5 minutes. This is possible due to the utilization of extremely high-speed electric currents as a means of creating cell death, whereas other ablation techniques use heat or cold temperature. A required temperature for RFA or Cryo is generated by ionic friction or sequential freezing and thawing of the tissues with mechanical energy, which require substantially increased ablation times (range, 15 to 60 minutes). ${ }^{19,20}$ In addition, any tumor larger than $3 \mathrm{~cm}$, or a tumor with an irregular shape will require additional ablations, which would require even more procedure time. This relatively short ablation time for IRE may correlate with reduced anesthesia time, reduced post-ablation pain, decreased ablation-related complications, decreased overall cost of ablation treatment, and as mentioned, it may provide an opportunity for treatment of multiple lesions or multiple treatments of single lesion in one session.

\section{Preservation of vital structures within IRE-ab- lated zone}

Another unique characteristic of IRE ablation that separates it from other ablative techniques is its capability of preserving vital structures within the IRE-ablated zone. In all IRE ablated liver tissues, critical structures, such as the hepatic arteries, hepatic veins, portal veins and intrahepatic bile ducts were all preserved. ${ }^{2}$ In other types of tumor ablation, these structures are completely obliterated due to the extreme temperature changes used by thermal ablation techniques, causing the denaturation of proteins. The denaturation process does not discriminate which proteins or DNA to destroy. Thus, all protein structures and cells with DNA can be damaged with the use of current thermal ablation techniques. However, with IRE ablation, cell death is mediated by apoptosis through disruption of the cell membrane. The structures mainly formed by proteins such as vascular elastic and collagenous structures, peri-cellular matrix proteins are not damaged by IRE ablation (Fig. 1). ${ }^{2,21}$ This leads to the preservation of structural scaffoldings of vessels and 

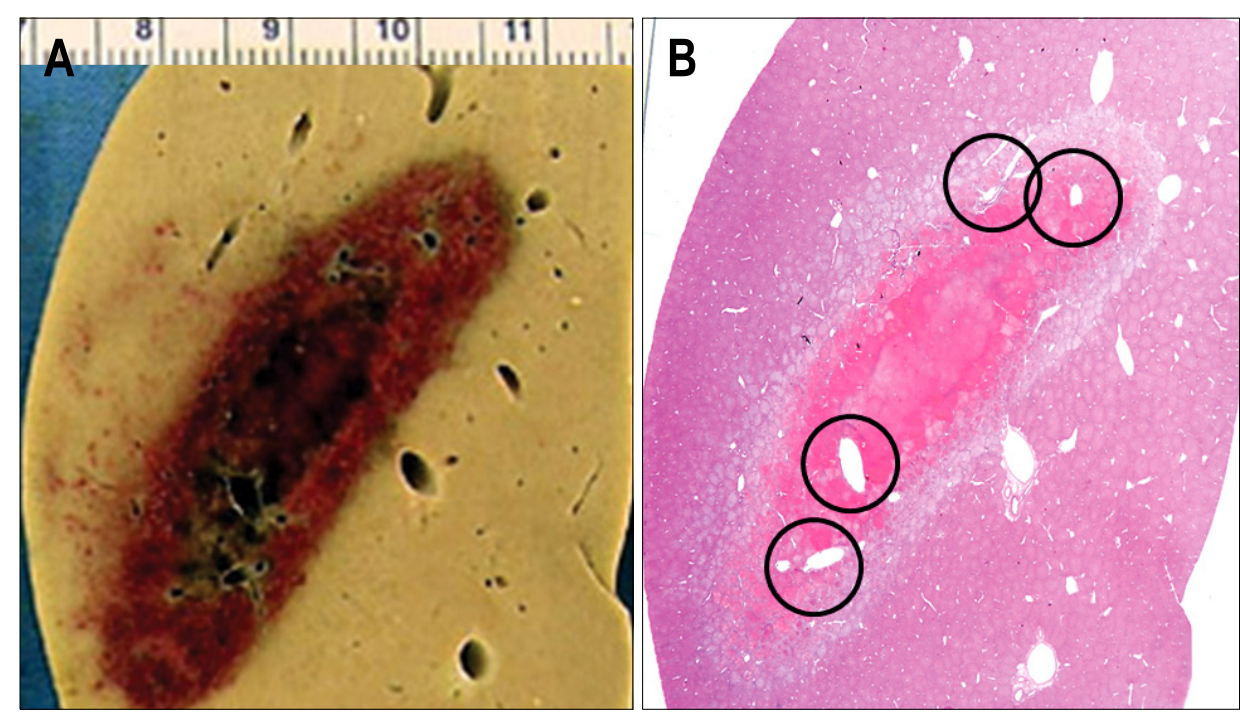

Fig. 1. Gross pathologic image (A) and hematoxylin and eosin (H\&E) staining (B) of irreversible electroporation (IRE) ablation zones show a sharp demarcation between the ablated and non-ablated, normal liver. Within the ablated zone, numerous vessels and bile ducts are observed as small as $1 \mathrm{~mm}$ in size with preserved structures.
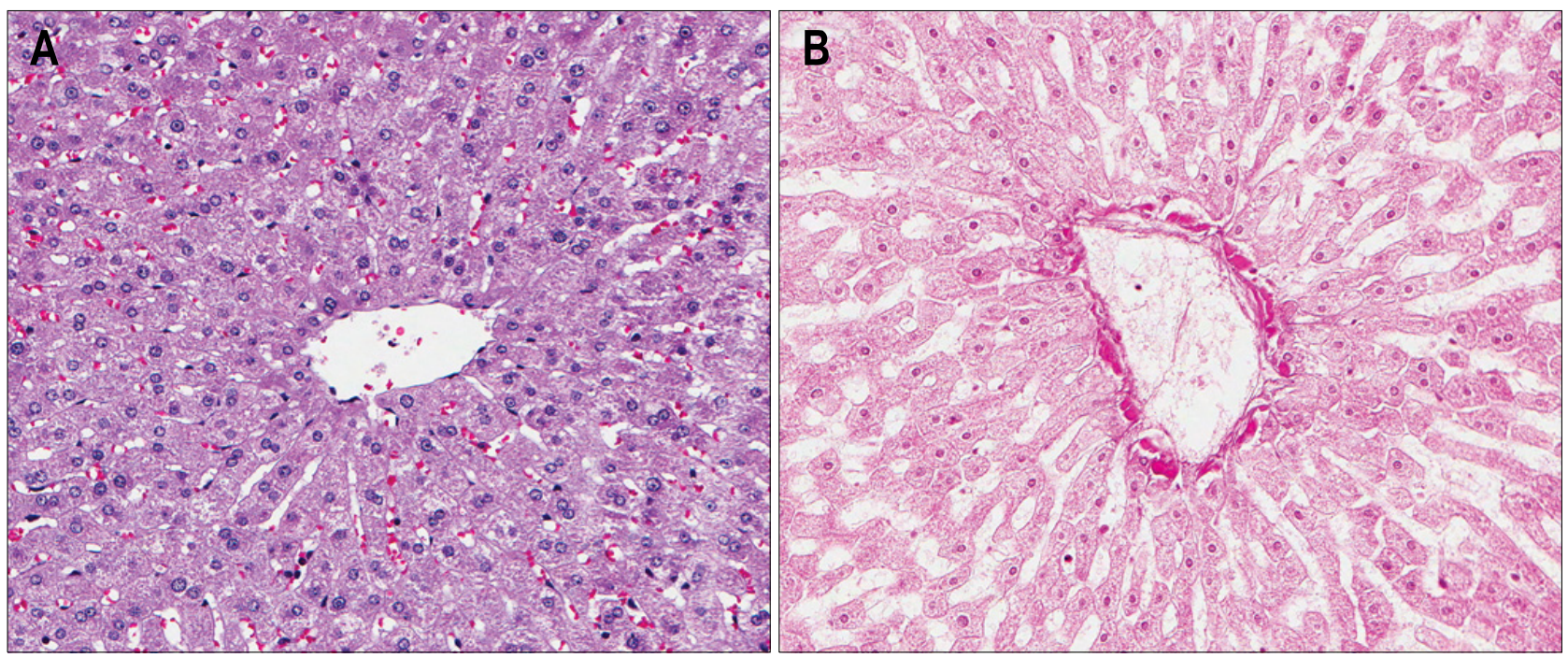

Fig. 2. Hepatocytes and central vein in the non-ablated zone (A) and irreversible electroporation (IRE)-ablated zone (B). All hepatocytes around the vessel in the IRE-ablated zone have been completely ablated and undergone cell death seen as pyknosis and karyorrhexis (H\&E stain, $\times 40)$.

bile ducts. The functional aspect of these structures may not be preserved at first due to lack of cellular functions. However, we hypothesize that the cellular functions also get restored as IRE-ablated tissues get regenerated. Currently, attempts are being made to further evaluate this regenerative function of IRE in tumor ablation.

\section{Avoidance of heat/cold-sink effect}

As mentioned above, one critical difference in IRE is its ability to create cell death via non-thermal ablation. A short, but powerful electric field (range, 1,500 to 3,000 volts) is applied to the targeted area and causes a disruption of cellular homeostasis through dismantling the cell membrane wall with innumerable nanopores. ${ }^{3,10}$ This disruption of cellular homeostasis causes apoptotic cell death and necrosis. ${ }^{2}$ The IRE treatment uses multiple ultra-short pulses which increases therapeutic effect and decreases thermal effects. This has been investigated and optimized by Davalos et al. ${ }^{9}$, who demonstrated that short pulses with brief intervals allow for cooling of tissues to avoid any thermal effect. As a result, the electric field created by IRE is devoid of any joule heating and therefore, non-thermal ablation is achieved. Since it does not depend on either heat or cold, IRE can create a focal tumor ablation area, independent of any heat or cold-sink effect. ${ }^{2}$ The heat-sink effect is created by the dissipation of abla- 
tive heat energy around a large vessel, ${ }^{22}$ and the cold-sink effect is a similar event where ablative freezing temperature is not reached around a large vessel. ${ }^{23}$ An important clinical implication is that the heat/cold-sink effect will cause incomplete ablation in the perivascular tumor cells and may result in tumor recurrence. Without being influenced by the heat or cold-sink effect, IRE can produce a more complete ablation within the lesion area, even with a large vessel traversing the ablation zone (Fig. 2).

\section{IRE-induced complete ablation with well-demar- cated margin}

Another notable benefit of IRE is its capability of creating complete cell death within the ablation area, regardless of the location, size and shape of the tumor. As described above, perivascular tumor cells and irregularly shaped or large sized tumors are completely ablated with IRE. In addition, all IRE ablated zones demonstrate sharp margins and sharp demarcation (on the order of 1-2 cell thickness) between the ablated areas and non-ablated areas. ${ }^{2}$ These findings are seen in both microscopic and macroscopic evaluations (Fig. 1). ${ }^{2,13,15}$ With its sharp demarcation, IRE ablation can be more accurately evaluated and assessed for effectiveness, tumor treatment outcome, and follow-up. Other thermal methods, such as RFA or MWA, create a "grey-zone" of ablation, where the most outer margin of ablation contains some living cells. Unlike RFA and MWA, IRE has no "grey-zone" of ablation. This "grey-zone" is significant because it is likely another source of incomplete ablation, causing a residual tumor or recurrence.

\section{IRE-induced apoptotic cell death}

Until recently, all tumor ablation was caused by coagulative necrosis, which creates unrecognizable tissue distortion and death in the area of thermal ablation. It seems as though coagulative necrosis is enough to kill all tumor cells, but it carries significant disadvantages as coagulative necrosis destroys cells unpredictably and in an uncontrolled manner. ${ }^{24,25}$ It destroys everything within the area of ablation, including vessels and bile ducts, leaving necrotic debris and dead tissues, which are toxic to the organs. In contrast, we have demonstrated apoptosis in IRE-induced cell death. ${ }^{2,13}$ Apoptosis is defined as physiological programmed cell death, occurring due to a wide variety of internal and external stimuli. ${ }^{24-26}$ Several different molecular, genetic, and protein markers are used to confirm apoptosis in various pathologic and oncologic processes. We have used BAX and TUNEL assays to confirm an element of apoptosis in IRE ablation (Fig. 3). The advantages of apoptotic cell death created by IRE include

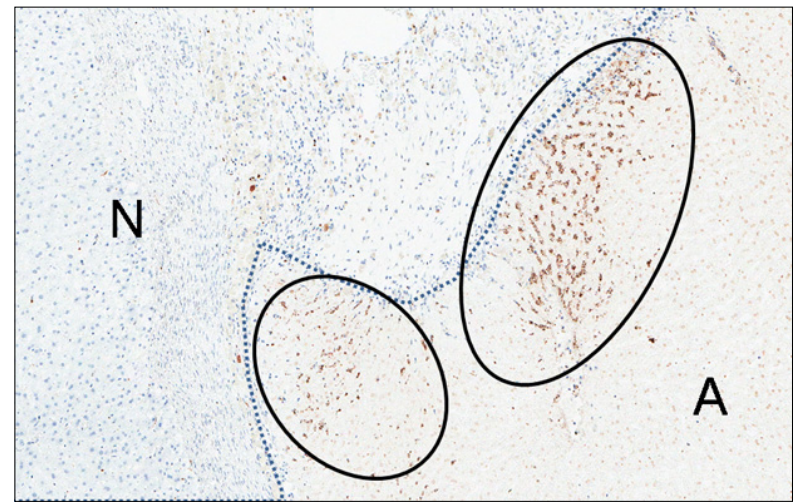

Fig. 3. High-magnification image of the irreversible electroporation (IRE)-ablated zone demonstrates normal liver tissue (N) without any TUNEL positive cells and markedly increased TUNEL positive cells seen in the IRE-ablated zone. An involvement of apoptosis has been confirmed by using multiple immunohistochemical assays including TUNEL assay, BCL-2 and Caspase staining.

the utilization of immune mediated cell death, which induces phagocytosis to clear up post-ablation debris, leading to fast recovery and regeneration by certain organs, since apoptosis is recognized as a natural course of each cell cycle.

\section{Real-time monitoring of IRE ablation}

Another clinical advantage of IRE ablation is the capability of using imaging guidance (US, CT, and MR) to monitor the procedure (Fig. 4). We are able to use imaging as guidance for the IRE probe placement, intra-ablation monitoring, and post-ablation monitoring of the treatment zone, which can be seen to be sharply demarcated on imaging in a similar fashion to that seen on histological data. ${ }^{2}$ The difference in size of the ablated zones monitored by US or CT versus gross pathological measurements of actual ablated zones were within a few millimeters, which confirms the accuracy of imaging in monitoring the extent of ablation. ${ }^{2}$ Any untreated residual tumor can be seen via imaging guidance to predict the need for additional IRE ablation to ensure each tumor is eradicated completely. In addition, real-time imaging monitoring can provide peri-ablation findings of patients to foresee any possibility of ablation related complications. This would also ensure the safety and rapid recovery of a patient after IRE ablation.

\section{MORE QUESTIONS REGARDING IRE}

Questions regarding the effects of strong electric fields created by IRE ablation on other non-targeted organs have surfaced. The first issue is muscle contraction due 

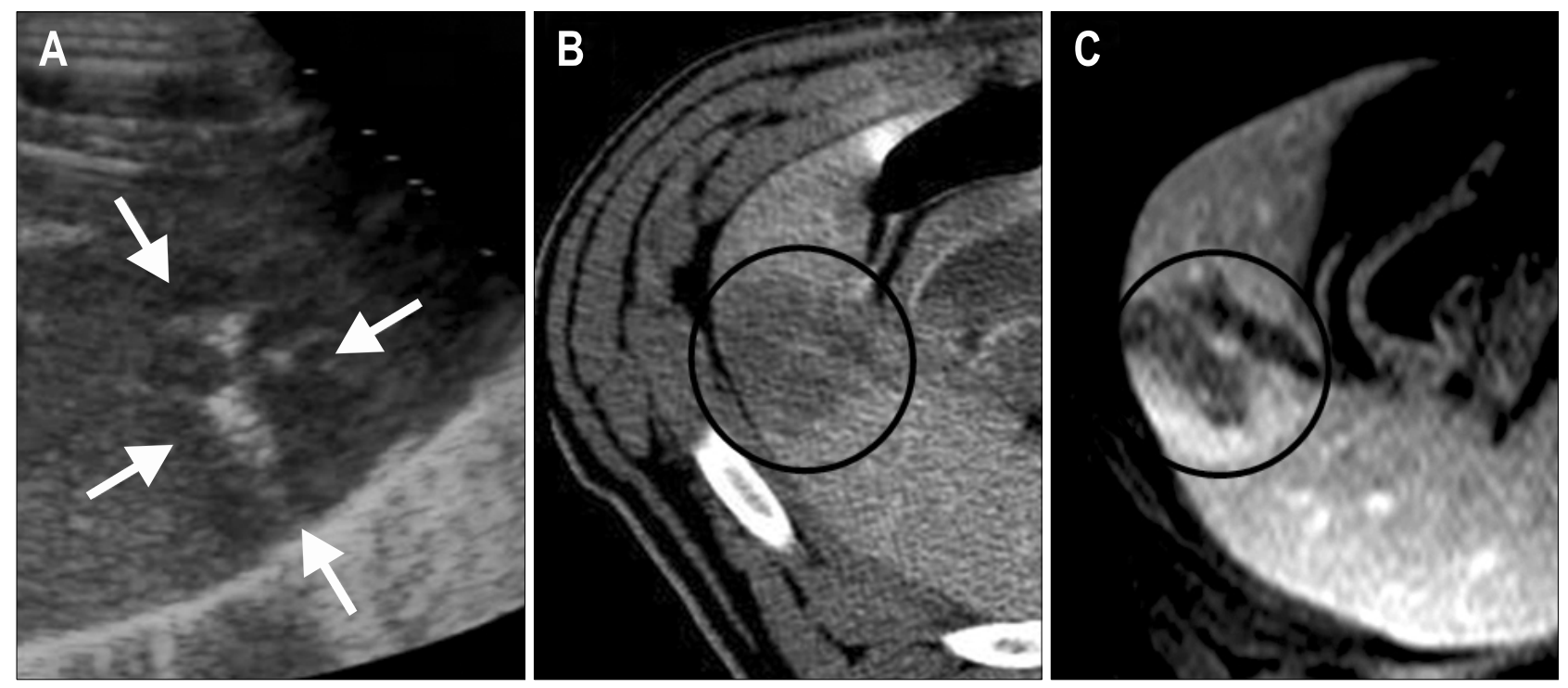

Fig. 4. Intra-procedure ultrasound image of irreversible electroporation (IRE) (A) demonstrates IRE's capability of real-time monitoring during and immediately after the procedure. A hypoechoic area of IRE ablation is observed with a sharp margin. Contrast-enhanced CT of the liver (B) in the delayed phase demonstrates a hypodense area of IRE ablation with increased contrast enhancement surrounding the ablated area. IRE-ablated area can be also well visualized and assessed using post-contrast fat-saturated T1-weighted MR image (C) demonstrating mildly increased enhancement in the area of ablation.

to IRE ablation. During pre-clinical research, prominent muscle contraction was observed in all animals treated with IRE. This muscle contraction was correlated with the power and length of the electric pulses created by IRE ablation. Therefore, shortening the pulse length and decreasing the amplitude of the pulse helped to decrease the muscle contraction. In addition, all current IRE ablations are performed using muscle relaxants, such as pancuronium in pre-clinical research, and cisatracurium in clinical settings.

The next issue is cardiac arrhythmias that can potentially be induced by IRE electrical pulses. It occurs most commonly in the "vulnerable" period of the cardiac cycle during atrial or ventricular systole. ${ }^{18,27,28}$ In addition, research subjects or patients with previously documented underlying structural or functional heart disease appeared to be at a greater risk for cardiac arrhythmia. No mortality has been associated with IRE-induced cardiac arrhythmia. Currently, all clinical IRE ablation units are accompanied with a cardiac synchronization device to ensure the safety of the patient. The cardiac synchronized IRE ablation can only produce pulses during the "non-vulnerable" period of the cardiac cycle. Since these synchronization devices have been developed, no further arrhythmias have been reported in clinical studies.

\section{CONCLUSION}

IRE is a new form of cancer treatment with the advantages of preserving important structures and organs, along with uniform and complete tumor destruction, an ultra-short ablation time, and real-time monitoring capability. More research is needed to look into areas of heterogeneous tissue ablation, and the underlying pathophysiology of irreversible electroporation needs to be illuminated. However, IRE proves to be promising for future clinical research and application.

\section{REFERENCES}

1. Daniels C, Rubinsky B. Electrical field and temperature model of nonthermal irreversible electroporation in heterogeneous tissues. J Biomech Eng 2009;131:071006.

2. Lee EW, Chen C, Prieto VE, Dry SM, Loh CT, Kee ST. Advanced hepatic ablation technique for creating complete cell death: irreversible electroporation. Radiology 2010;255: 426-433.

3. Rubinsky B, Onik G, Mikus P. Irreversible electroporation: a new ablation modality--clinical implications. Technol Cancer Res Treat 2007;6:37-48.

4. Rubinsky B. Irreversible electroporation in medicine. Technol Cancer Res Treat 2007;6:255-260.

5. Fuller GW, Louisville Water Company (Louisville Ky.). Report on the investigations into the purification of the Ohio River water: at Louisville, Kentucky, made to the president and directors of the Louisville Water Company. New York: D. Van Nostrand Company, 1898. 
6. Rockwell $\mathrm{AD}$. The medical and surgical uses of electricity, including the X-ray, Finsen light, vibratory therapeutics, and high-frequency currents. New ed. New York: E. B. Treat \& Company, 1903.

7. Neumann E, Schaefer-Ridder M, Wang Y, Hofschneider $\mathrm{PH}$. Gene transfer into mouse lyoma cells by electroporation in high electric fields. EMBO J 1982;1:841-845.

8. Wong TK, Neumann E. Electric field mediated gene transfer. Biochem Biophys Res Commun 1982;107:584587.

9. Davalos RV, Mir IL, Rubinsky B. Tissue ablation with irreversible electroporation. Ann Biomed Eng 2005;33:223231.

10. Edd JF, Horowitz L, Davalos RV, Mir LM, Rubinsky B. in vivo results of a new focal tissue ablation technique: irreversible electroporation. IEEE Trans Biomed Eng 2006;53: 1409-1415.

11. Onik G, Mikus P, Rubinsky B. Irreversible electroporation: implications for prostate ablation. Technol Cancer Res Treat 2007;6:295-300.

12. Al-Sakere B, Andre F, Bernat C, et al. Tumor ablation with irreversible electroporation. PLoS ONE 2007;2:e1135.

13. Lee EW, Loh CT, Kee ST. Imaging guided percutaneous irreversible electroporation: ultrasound and immunohistological correlation. Technol Cancer Res Treat 2007;6:287294.

14. Choi YS, Kim HB, Chung J, Kim HS, Yi JH, Park JK. Preclinical analysis of irreversible electroporation on rat liver tissues using a microfabricated electroporator. Tissue Eng Part C Methods. Forthcoming 2010. DOI: 10.1089/ ten.tec.2009.0803.

15. Guo Y, Zhang Y, Klein R, et al. Irreversible electroporation therapy in the liver: longitudinal efficacy studies in a rat model of hepatocellular carcinoma. Cancer Res 2010;70: 1555-1563.

16. Ellis TL, Garcia PA, Rossmeisl JH, Henao-Guerrero N, Robertson J, Davalos RV. Nonthermal irreversible electroporation for intracranial surgical applications. J Neurosurg. Forthcoming 2010. DOI: 10.3171/2010.5.JNS091448.

17. Neal RE 2nd, Singh R, Hatcher HC, Kock ND, Torti SV, Davalos RV. Treatment of breast cancer through the appli- cation of irreversible electroporation using a novel minimally invasive single needle electrode. Breast Cancer Res Treat 2010;123:295-301.

18. Ball C, Thomson KR, Kavnoudias H. Irreversible electroporation: a new challenge in "out of operating theater" anesthesia. Anesth Analg 2010;110:1305-1309.

19. Goldberg SN, Solbiati L, Hahn PF, et al. Large-volume tissue ablation with radio frequency by using a clustered, internally cooled electrode technique: laboratory and clinical experience in liver metastases. Radiology 1998;209:371379.

20. Solbiati L, Ierace $\mathrm{T}$, Tonolini M, Osti V, Cova L. Radiofrequency thermal ablation of hepatic metastases. Eur J Ultrasound 2001;13:149-158.

21. Maor E, Rubinsky B. Endovascular nonthermal irreversible electroporation: a finite element analysis. J Biomech Eng 2010;132:031008.

22. Lu DS, Raman SS, Vodopich DJ, Wang M, Sayre J, Lassman C. Effect of vessel size on creation of hepatic radiofrequency lesions in pigs: assessment of the "heat sink" effect. AJR Am J Roentgenol 2002;178:47-51.

23. Bhardwaj N, Strickland AD, Ahmad F, Atanesyan L, West $\mathrm{K}$, Lloyd DM. A comparative histological evaluation of the ablations produced by microwave, cryotherapy and radiofrequency in the liver. Pathology 2009;41:168-172.

24. Kerr JF, Wyllie AH, Currie AR. Apoptosis: a basic biological phenomenon with wide-ranging implications in tissue kinetics. Br J Cancer 1972;26:239-257.

25. Thompson CB. Apoptosis in the pathogenesis and treatment of disease. Science 1995;267:1456-1462.

26. Susin SA, Lorenzo HK, Zamzami N, et al. Molecular characterization of mitochondrial apoptosis-inducing factor. Nature 1999;397:441-446.

27. Jones M, Geddes LA. Strength-duration curves for cardiac pacemaking and ventricular fibrillation. Cardiovasc Res Cent Bull 1977;15:101-112.

28. Lavee J, Onik G, Mikus P, Rubinsky B. A novel nonthermal energy source for surgical epicardial atrial ablation: irreversible electroporation. Heart Surg Forum 2007;10: E162-E167. 